\title{
Que nous apprennent les brevets de Tesla Motors sur sa stratégie PI?
}

\section{What can we learn from Tesla Motor's patents about its IP strategy?}

\author{
Marc Baudry ${ }^{1}$, Béatrice Dumont ${ }^{2}$ \\ ${ }^{1}$ Université Paris Nanterre, EconomiX-CNRS \& Chaire Economie du Climat, marc.baudry@parisnanterre.fr \\ ${ }^{2}$ Université Sorbonne Paris Nord, CEPN-CNRS \& College of Europe, beatrice.dumont@univ-paris13.fr
}

\begin{abstract}
RÉSUMÉ. Le patent pledge de l'entreprise Tesla est un cas d'étude particulièrement intéressant. II correspond en effet à une stratégie d'ouverture de son portefeuille de brevets par une entreprise nouvelle, qui a investi dans un secteur déjà fortement concentré en ciblant un marché spécifique, celui du véhicule tout électrique, par le haut de gamme. II ressort de l'analyse des brevets que Tesla est tout à la fois très peu engagé dans les coopérations de R\&D en amont, au moins tel qu'il est possible d'en juger par les co-brevets de la firme en comparaison avec ceux de ses concurrents, et au cœur des classes technologiques où il brevète, à en juger par le nombre significativement plus élevé de citations reçues par ses brevets. Ces éléments arguent en faveur d'une interprétation en termes de recherche de leadership technologique par Tesla. Cette interprétation est confortée par l'intégration verticale de Tesla. Selon cette interprétation, le patent pledge est un moyen d'amener les concurrents à s'aligner sur les solutions techniques proposées par Tesla afin pour ce dernier de tirer parti d'externalité de réseau et d'économies d'échelle et de baisser ainsi ses coûts pour imposer sa technologie sur le marché des véhicules tout électriques.

ABSTRACT. The Tesla patent pledge is a particularly interesting case study. It corresponds to a strategy of opening up its patent portfolio by a new company, which has invested in an already highly concentrated sector by targeting a specific market, that of the all-electric vehicle, by the top of the range. The analysis of the patents shows that Tesla is both very little involved in upstream R\&D cooperation, at least as far as it is possible to judge by the firm's co-patents compared to those of its competitors, and at the heart of the technological classes where it patents, judging by the significantly higher number of citations received by its patents. These elements argue in favour of an interpretation in terms of Tesla's search for technological leadership. This interpretation is supported by Tesla's vertical integration. According to this interpretation, the patent pledge is a means of bringing competitors into line with Tesla's technical solutions in order for Tesla to take advantage of network externalities and economies of scale and thus lower its costs to impose its technology on the market for all-electric vehicles.

MOTS-CLÉS. Tesla, Voiture électrique, Innovation de disruption, co-opétition en R\&D.

KEYWORDS. Patent pledge, Tesla, Electric cars, Disruptive innovation, Co-opetition in R\&D.
\end{abstract}

\section{Introduction}

La firme californienne Tesla est connue pour bousculer les habitudes du secteur automobile [THO 19]. Elle a d'abord eu l'audace de pénétrer le marché automobile, malgré l'importance des barrières à l'entrée, pour se lancer dans la production de véhicules tout électriques à partir d'une technologie obtenue sous licence auprès de l'entreprise AP propulsion. Elle l'a en outre fait en s'attaquant au haut de gamme, là où les tentatives précédentes avaient visé le bas de gamme. De plus, aux EtatsUnis, elle tend à remettre en cause la réglementation interdisant aux constructeurs de disposer de leur propre réseau de distribution ([BAR 14], [STO 15], [CRA 16]). L'entreprise Tesla ne s'est pas contentée de fabriquer des voitures. Elle a aussi adopté une stratégie d'intégration verticale, à l'encontre de ce qui se fait dans le secteur automobile, en développant son propre système de recharge, et en le couplant à une solution de production et de stockage d'électricité d'origine renouvelable par les clients, le powerwall. Finalement, son Directeur Général Elon Musk annonçait très médiatiquement le 12 juin 2014 au nom de la société qu'il dirige «All our patent are belong to you». Dans le même communiqué Elon Musk allait jusqu'à remettre en cause une stratégie d'innovation construite sur les brevets : «Technology leadership is not defined by patents, which

\footnotetext{
${ }^{1}$ Consultable en ligne à l'adresse https://www.tesla.com/blog/all-our-patent-are-belong-you?redirect=no
} 
history has repeatedly shown to be small protection indeed against a determined competitor, but rather by the ability of a company to attract and motivate the world's most talented engineers. ». Dans ce même communiqué, Elon Musk annonçait que Tesla mettait dorénavant à disposition de tous ses concurrents ses brevets, sans contrepartie si ce n'est qu'ils se comportent de manière «juste» («fair»). Tesla a par la suite précisé son intention, tant au sujet de cette notion de comportement «juste» qu'au sujet des brevets mis à disposition et de la durée de son engagement ${ }^{2}$. Si elle l'a sans doute fait avec une stratégie de communication bien pensée, Tesla n'est ni la première ni la dernière firme à annoncer ouvrir ainsi son portefeuille de brevets dans le cadre de ce qui appelé un patent pledge.

On désigne sous le terme patent pledge l'engagement d'un détenteur de brevet d'amoindrir leur assertion par rapport à ce qui est légalement possible [CON 15]. Il s'agit essentiellement d'autoriser, à différents degrés, l'exploitation du brevet par d'autres que son propriétaire. Cette liberté peut être limitée à quelques brevets et avec un principe de réciprocité dans le cadre de licence gratuites croisées, ou être limitée dans le temps et selon le type de brevets comme dans le cas du patent pledge de Toyota sur la technologie de la pile à hydrogène, ou bien encore concerner tout le portefeuille sans contrepartie financière ou non financière (par exemple la réciprocité) et sans limite de temps comme dans le patent pledge de Tesla. Un patent pledge n'est généralement pas désintéressé. S'il l'était totalement, le détenteur de brevets pourrait plus simplement annoncer laisser tomber dans le domaine public ses brevets. De ce point de vue, ce qui attire l'attention sur le cas du patent pledge de Tesla, outre la relative habileté de communication qui l'a accompagné et la grande liberté d'exploitation accordée aux concurrents, c'est aussi le plaidoyer contre le système des brevets qui l'a accompagné. Sur les blogs spécialisés en Propriété Intellectuelle ou dans la presse économique, les commentaires ont pu se montrer très dubitatifs, voire critiques quant à l'apparente philanthropie de l'engagement pris par Tesla ${ }^{3}$.

Cet article propose donc d'analyser la décision de Tesla de manière objective et en la contextualisant. Il est étayé à partir de données de brevets extraites de la base Patstat puis traitées par les auteurs ${ }^{4}$. Ainsi, une première section dresse un état des lieux de la position de Tesla sur le marché des véhicules électriques, mais compare aussi la firme aux autres du secteur en termes de taille du portefeuille de brevets. La seconde section cherche à évaluer s'il y a une originalité de Tesla en matière de brevets. Elle cherche plus particulièrement à tester dans quelle mesure il y a eu rupture ou non dans la stratégie de dépôt de brevets de Tesla à partir de l'annonce de son patent pledge de 2014. Elle vise également à comparer la stratégie d'internationalisation de son portefeuille de brevets par rapport à ses concurrents. La troisième section s'interroge sur la stratégie en termes de coopérations de R\&D qu'a pu mettre en place Tesla, en l'évaluant notamment à l'aune des cobrevets et en la comparant également à celle de ses concurrents. Elle amène à suggérer que le patent pledge de Tesla est destiné à transformer un certain leadership technologique en leadership sur le marché, ce qui est conforté à partir d'un examen des citations de brevets dans la quatrième section. Cette dernière section propose également un décryptage de la rationalité économique sous-jacente à la l'engagement de Tesla à l'aide des concepts d'externalités de réseaux et d'économies d'échelle.

\section{Tesla, un petit dans la cour des grands?}

Si Tesla marque les esprits, c'est parce qu'il s'agit d'un des rares constructeurs nouveaux ayant réussi à s'imposer durablement dans un secteur marqué par une tendance à la concentration, notamment aux Etats-Unis. Ce succès, Tesla le doit à sa stratégie de différenciation centrée sur la

\footnotetext{
${ }^{2}$ Voir https://www.tesla.com/about/legal\#patent-pledge

${ }^{3}$ Voir par exemple le blog de IPwatchdog, notamment le billet https://www.ipwatchdog.com/2014/07/08/tesla-on-patents-open-sourcealtruism-or-shrewd-business/id=50331/

${ }^{4}$ Voir https://www.epo.org/searching-for-patents/business/patstat.html\#tab-1

(c) 2020 ISTE OpenScience - Published by ISTE Ltd. London, UK - openscience.fr 
vente, hors réseau commercial standard, de véhicules purement électriques positionnés sur le haut de gamme. Dès lors, comparer Tesla aux autres constructeurs est délicat. Ses volumes de ventes en 2018 ne dépassent pas les 300000 unités alors qu'il est considéré dans le secteur qu'il faut atteindre dans les quatre millions d'unités pour rentabiliser les investissements, notamment ceux en R\&D requis pour relever les défis du véhicule du futur. L'exercice de comparaison requiert donc, dans un premier temps, d'identifier à qui comparer Tesla. La solution retenue ici est celle d'une approche globale de l'ensemble des constructeurs ou marques. Elle se justifie par deux arguments. Le premier est que le défi du développement électrique, dans une perspective de transition énergétique pour répondre au réchauffement climatique, est planétaire. Le second est que Tesla a bousculé ou inspiré de nombreux constructeurs à travers le monde. C'est le cas des constructeurs premium allemands comme Mercedes Benz, BMW, Audi, Porsche qui ont tous aujourd'hui au moins un modèle tout électrique dans leur gamme après avoir laissé Tesla faire cavalier seul pendant plusieurs années. Plus récemment, des constructeurs de gamme intermédiaire comme Renault ou PSA en France s'y sont également mis. Sur le marché américain, General Motors (GM) a aussi tenté d'être disruptif avec la Volt, également commercialisée en Europe sous la marque Opel et le nom Ampera avant le rachat de ce dernier par PSA ${ }^{5}$. Les constructeurs asiatiques ne sont pas en reste, tant au Japon, en Corée qu'en Chine. On retrouve par exemple en Chine le constructeur BYD spécialisé dans le tout électrique. On notera également que le développement de Tesla cible tout particulièrement le marché chinois avec la construction d'une grande usine à Shanghaï.

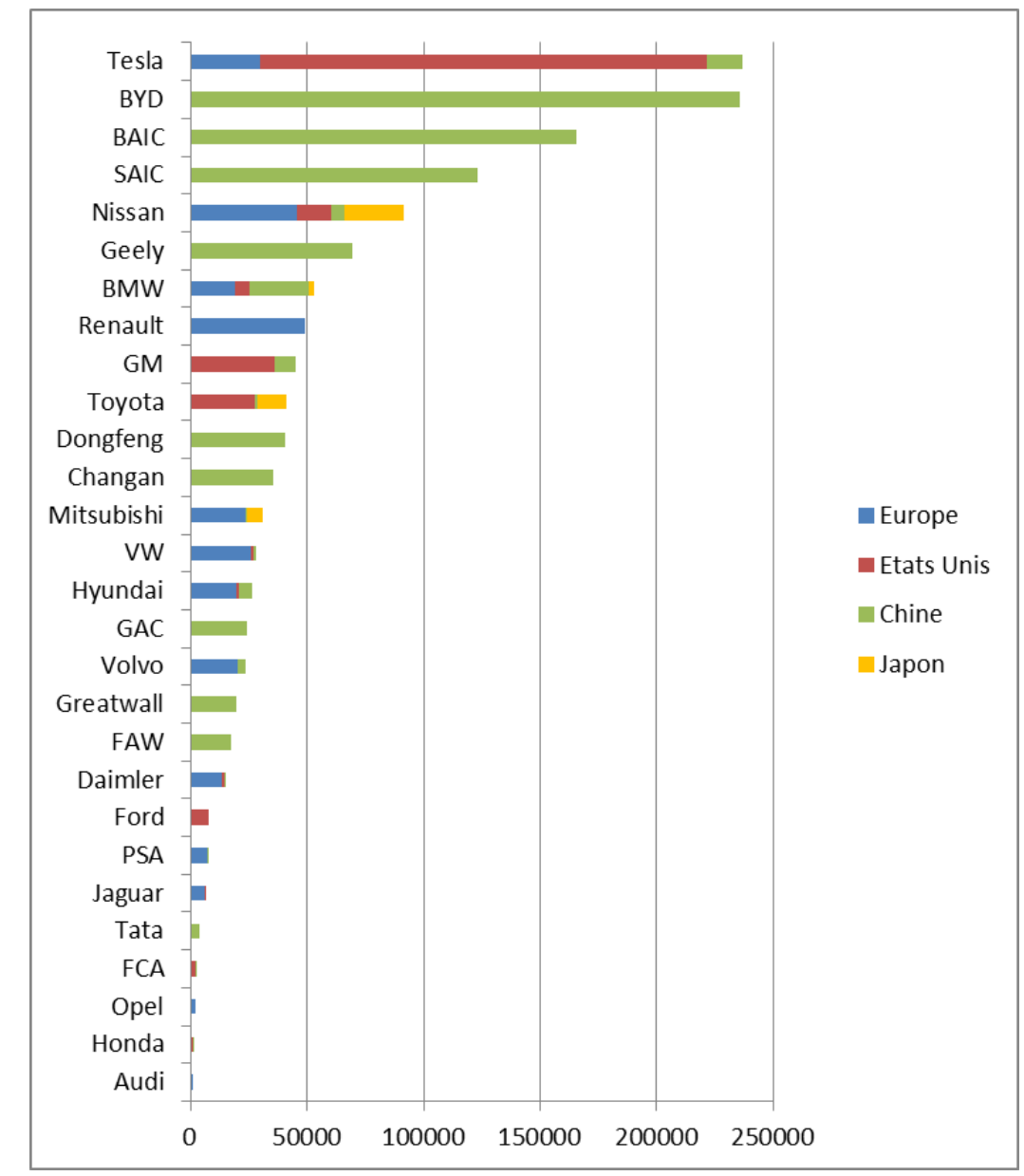

Figure 1. Nombre de ventes de véhicules tout électrique à batterie en 2018 selon le constructeur et la zone géographique.

Source : Statista complété par le site INSIDEEVs

\footnotetext{
${ }^{5}$ Il est intéressant à ce sujet de noter que les brevets sur ce véhicule étant déposés au nom de GM, PSA ne peut que les exploiter sur le modèle Ampera tant que celui-ci continue à être commercialisé par Opel.
} 
La Figure 1 donne une idée de l'état de la concurrence opposée à Tesla en 2018 sur le seul segment des véhicules tout électrique à batterie ${ }^{6}$, en se concentrant sur les zones géographiques où le marché a une taille significative (Europe, Etats-Unis, Chine, Japon). Tesla était en 2018 leader au niveau mondial, mais pas nécessairement sur chaque zone géographique. Le marché chinois est par exemple largement dominé par des constructeurs chinois ayant bénéficié d'une politique volontariste des autorités locales, sous formes de subventions publiques. De même en Europe, Renault et Nissan (présentés séparément mais liés par une alliance et offrant des véhicules tout électriques conçus sur une même plateforme et technologie) devancent Tesla, et de nombreux autres constructeurs européens (BMW, Volkswagen, Volvo) ou asiatiques (Mitsubishi, Hyundai) le talonnent. C'est d'abord et avant tout sur le marché américain que Tesla domine largement ses concurrents.

Tesla s'est démarqué par son approche disruptive dans le développement, mais aussi la commercialisation de véhicules entièrement électriques. Cette approche disruptive est valorisée par les marchés, si on se fie à la capitalisation boursière rapportée aux volumes de vente ${ }^{7}$, mais le défi du passage à la production industrielle de grands volumes est régulièrement pointé du doigt. Le caractère disruptif de la stratégie de Tesla pourrait suggérer un bon positionnement de la firme par rapport aux autres constructeurs en matière d'innovation et, plus singulièrement, pour un secteur caractérisé par une rétro-ingénierie relativement facile, un bon positionnement en termes de taille du portefeuille de brevets. Mais ce serait oublier que ce caractère disruptif amène précisément Tesla sur des champs technologiques potentiellement assez différents de ceux des autres constructeurs. Tesla pourrait donc dominer technologiquement avec un portefeuille de brevets finalement assez limité. Un premier examen des tailles de portefeuilles de brevets s'impose donc. La période étudiée va de 2006, date à laquelle Tesla Motors a déposé son premier brevet selon la base Patstat, jusqu'à 2018 . L'année 2019 est trop récente pour que, au moment de la collecte des données la base Patstat soit exhaustive pour cette année. La Figure 2 positionne Tesla par rapport à une liste de concurrents, plus large que la liste de seuls concurrents, recensés à la Figure 1, offrant également dans leur catalogue un véhicule tout électrique. Cette liste appelle un commentaire: Sont recensés tantôt des constructeurs, tantôt des marques. Le choix s'est fait au cas par cas, selon que les marques d'un même constructeur témoignaient d'une activité de dépôt de brevets significative et donc, a priori, d'une stratégie d'innovation qui pouvait leur être spécifique. C'est ainsi que pour le groupe VAG sont distinguées les trois marques Volkswagen, Audi et Porsche, les autres marques (par exemple Skoda ou Seat) n'ayant pas une activité suffisamment forte. Des marques qui ont changé de groupe au cours de la période étudiée ont également été traitées séparément. C'est par exemple le cas de la marque suédoise Volvo passée du giron de l'américain Ford à celui du chinois Geely. C'est également le cas du germano anglais Opel/Vauxhaul passé sous contrôle du français PSA après avoir longtemps dépendu de l'américain GM. Certaines entités crées ex nihilo, suite à fusion ou rachat sont également traitées à part des marques concernées par l'opération de fusion ou acquisition. C'est le cas typique de FCA qui a une activité de dépôt de brevets en parallèle aux marques Chrysler et Fiat. A contrario, certains constructeurs qui disposent de plusieurs marques

\footnotetext{
${ }^{6}$ Les véhicules hybrides, également offerts par la plupart des concurrents de Tesla, sont exclus car ils ne présentent pas les mêmes avantages et défauts que les véhicules tout électriques à batteries et correspondent à un marché différent. De même, les véhicules électriques fonctionnant à partir d'une pile à combustible (notamment à base d'hydrogène) ne sont pas retenus dans ces statistiques. Ces véhicules sont encore très peu diffusés mais pourraient à terme constituer une concurrence très sérieuse à Tesla car combinant le caractère « zéro émissions de $\mathrm{CO} 2$ » du tout électrique (sous réserve que l'hydrogène, comme l'électricité des véhicules tout électriques, soit elle-même produite de manière « propre ») et l'autonomie et la rapidité de recharge des véhicules à moteur thermique.

7 "Tesla a dépassé pour la première fois le 22 janvier 2020 le seuil symbolique de 100 milliards de dollars de capitalisation boursière, surclassant Volkswagen et talonnant Toyota. (...) Autrement dit en ordre de grandeur, Tesla vaut désormais cinq fois PSA tout en produisant dix fois moins de voitures... » (source, La Tribune, disponible à l'adresse https://www.latribune.fr/entreprises-finance/industrie/automobile/teslafranchit-les-100-milliards-de-dollars-de-capitalisation-en-bourse-837798.html.
} 
déposent essentiellement leurs brevets sous le nom du groupe plutôt que des marques, tel PSA qui dispose des marques Peugeot, Citroën, DS, et maintenant également Opel.

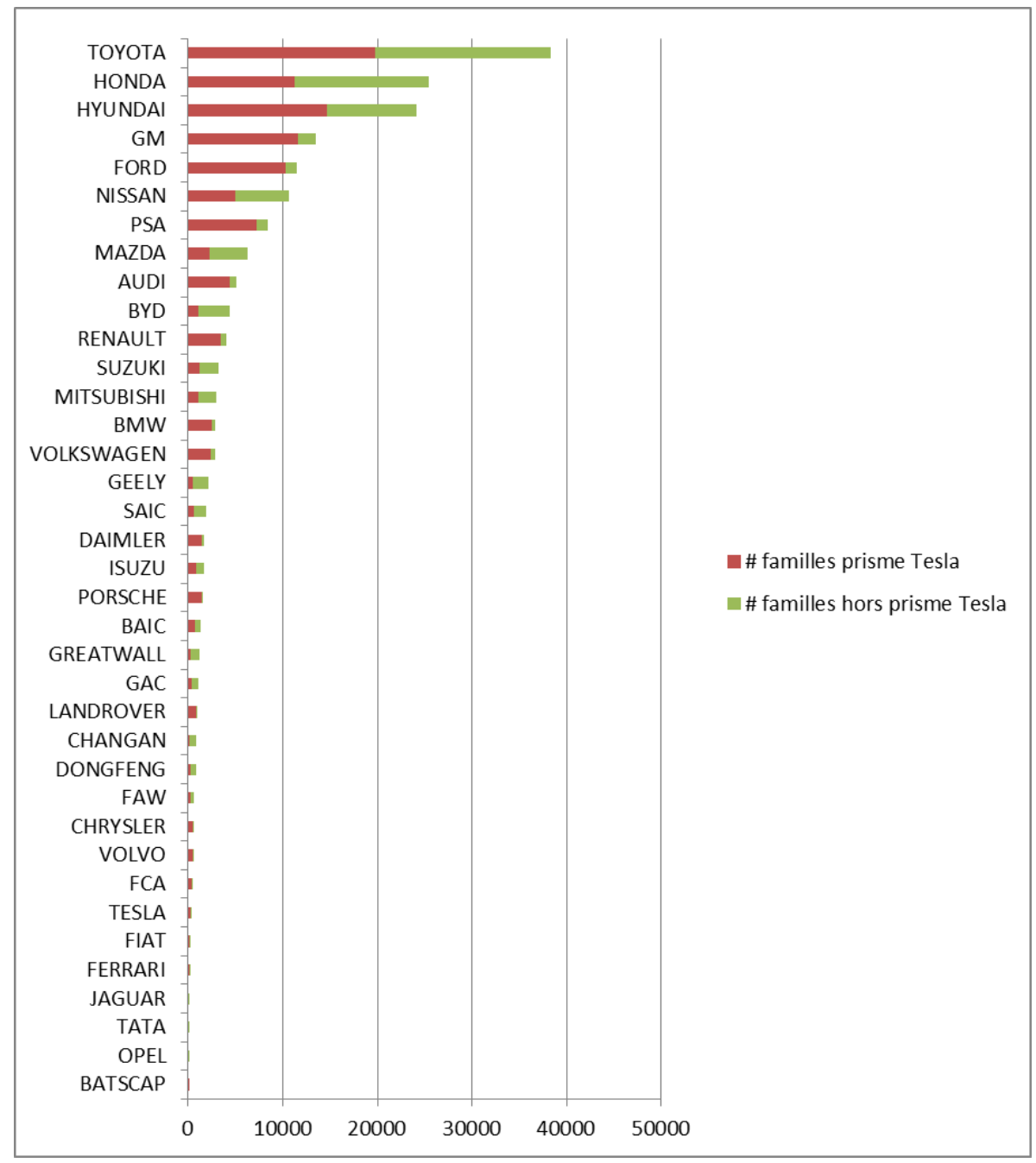

Figure 2. Taille du portefeuille des constructeurs automobiles en termes de nombre de familles et décomposition entre familles ciblant des classes technologiques ciblées par Tesla et familles ne ciblant aucune des classes technologiques ciblées par Tesla (période 2006-2018)

Source : auteurs à partir d'extractions de Patstat

L'exercice de comparaison des tailles de portefeuille de brevets illustré par la Figure 2 a privilégié l'approche par les familles de brevets. Il faut à ce stade rappeler qu'une famille regroupe l'ensemble des brevets déposés auprès des différents offices de brevets de par le monde et portant sur une même invention. Ces familles sont construites sur la base des priorités inscrites dans le document brevet, c'est-à-dire de quels autres brevets ils considèrent être l'extension auprès d'un nouvel office, ou parfois au sein du même office ${ }^{8}$. Travailler sur les familles revient donc à se concentrer sur le dénombrement des inventions. Le nombre de brevets dans la famille (appelé taille de la famille) peut être un indicateur de la valeur de l'invention, le déposant cherchant à couvrir une aire géographique d'autant plus large qu'il pense que l'invention protégée est amenée à une industrialisation et une commercialisation sur des marchés variés à l'international. La Figure 2 met clairement en évidence que Tesla reste un petit dans la cour des grands, y compris en termes de nombre d'inventions. Beaucoup de constructeurs inconnus en Europe ou en Amérique de Nord,

\footnotetext{
${ }^{8}$ Il existe néanmoins différentes façons de construire une famille. La base Patstat en propose deux, les familles INPADOC et les familles DOCDB. C'est sur ces dernières que l'étude a porté.
} 
notamment des constructeurs chinois guère plus anciens que Tesla, ont un portefeuille d'inventions plus large. Le leadership, tel qu'appréhendé par ces premières statistiques, est représenté par Toyota, suivi de plusieurs constructeurs également Japonais, ou Coréen. Ce classement est bien entendu perfectible. Le rang du groupe VAG est par exemple minimisé par la distinction entre trois de ses marques. On sait également que la limitation du nombre de revendications dans les brevets japonais conduit à fragmenter auprès du JPO une demande sur une même invention en de multiples brevets. De même, des interrogations importantes existent quant à la qualité des brevets déposés auprès du CNIPA (ex SIPO) ; le cadre institutionnel chinois ayant favorisé une inflation des dépôts de brevets en grande partie déconnectée de l'évolution des dépenses de R\&D en Chine. Il est toutefois douteux que la correction de ces différents éléments conduise à un diagnostic radicalement différent quant à la taille réduite de Tesla en termes de portefeuille d'innovation. Ceci est d'autant plus probable que, si on restreint le comptage des familles de brevets aux seules familles dont au moins un brevets cible l'une ou l'autre des classes technologiques ciblées par les brevets de Tesla, les ordres de grandeur des portefeuilles ne sont pas pour autant fondamentalement changés ${ }^{9}$. Autrement dit, même en se restreignant aux champs technologiques de prédilection de Tesla, celui reste un petit acteur en termes de taille de portefeuille d'inventions ${ }^{10}$. Une conséquence pour la suite de l'analyse est qu'il faut prendre la précaution de vérifier qu'il n'y a pas un « effet taille » dans l'analyse de la stratégie de Tesla. La Figure 2 illustre en outre de manière frappante que, même en se restreignant aux seules familles qui ciblent au moins une des classes ciblées par les brevets de Tesla, la firme californienne est de très loin dominée par nombre d'autres constructeurs. Cela signifie que si le modèle d'affaire global de Tesla est original et atypique, ce n'est pas nécessairement le cas du type d'innovation technologique sur lequel il s'appuie.

\section{Tesla a-t-il réellement une stratégie PI originale?}

Au-delà de la taille du portefeuille de brevets, le patent pledge de Teslas suggère, au moins tel que formulé dans l'annonce officielle faite par Elon Musk, une rupture dans la stratégie PI de Tesla. Plus précisément, cette annonce a laissé entendre l'abandon de la protection des inventions par la société au moyen des brevets. Les commentateurs ont discuté la réalité de cet engagement, suggérant qu'il s'agissait plus d'une ouverture du portefeuille de brevet, avec éventuellement un système de licence de type FRAND (Fair, Reasonable, and non-Discriminatory), voire de licences gratuites, mais pas d'un abandon de la protection par la PI. Le recul temporel par rapport à cette annonce est aujourd'hui suffisamment important pour l'aborder au travers d'éléments factuels tangibles. Les Figures 3.a et 3.b comparent notamment le flux de nouveaux membres dans le portefeuille de familles DOCDB pour Tesla et pour les autres constructeurs. Il est indispensable de procéder par comparaison plutôt que d'examiner en absolu ce flux afin d'éviter certains écueils. En effet, afin de s'assurer que les inventions intégrées à l'étude remplissent bien les critères de brevetabilité, l'analyse a été limitée aux brevets octroyés, et non pas juste aux demandes déposées. De ce fait, compte tenu des délais d'examen par les offices, de nombreuses demandes formulées récemment n'ont très probablement pas encore fait l'objet d'une décision. Il en résulte une baisse « technique » du nombre de brevets, et donc des familles, nouvellement recensés sur les années les

\footnotetext{
${ }^{9}$ Les classes technologiques en question sont celles de la Cooperative Patent Classification (CPC) telles que déclarées dans les documents de brevets, et donc enregistrées sur Patstat. C'est le niveau 4 digits de la classification CPC qui a été utilisé dans toute cette étude. Le coefficient de corrélation entre le nombre de familles ciblant les mêmes classes que Tesla et le nombre de familles ne ciblant aucune des classes ciblées par Tesla est de 0.8168 sur l'ensemble des constructeurs ou marques intégrés à l'étude.

${ }^{10}$ Les classes CPC ciblées par Tesla sur la période étudiée sont les suivantes (classes CPC à 4 digits) : B01D, B04C, B08B, B21C, B21D, B23K, B23P, B25J, B29C, B29L, B32B, B60G, B60H, B60J, B60K, B60L, B60N, B60Q, B60R, B60S, B60T, B60W, B60Y, B62D, B67D, C09J, E05B, E05C, E05D, E05F, E05Y, E06B, F01M, F04B, F16B, F16F, F16H, F16K, F28D, F28F, F41H, G01C, G01J, G01K, G01R, G02B, G05D, G06F, G06Q, G07C, G08B, H01B, H01F, H01G, H01H, H01L, H01M, H01R, H02B, H02G, H02H, H02J, H02K, H02M, H02P, H03K, H04L, H04M, H04Q, H04R, H04S, H04W, H05K, Y02B, Y02D, Y02E, Y02T, Y04S, Y10S, Y10T.
} 
plus récentes (allant jusqu'à zéro pour l'année 2018). Ceci quel que soit le déposant considéré. Les comparaisons effectuées aux Figures 3.a et 3.b sont en outre facilitées de deux manières. La première manière consiste en une neutralisation des différences de taille dans les flux par la normalisation de ces tailles à un pour l'année 2006. La valeur portée sur l'axe vertical se lit donc comme le facteur multiplicatif à appliquer par rapport au flux observé en 2006. La seconde manière consiste à construire un intervalle de valeurs «standards », en grisé, indiquant la dispersion des autres constructeurs autour du flux moyen calculé pour ces derniers ${ }^{11}$. Toute sortie par le haut (respectivement par le bas) de la zone grisée par la courbe de flux de Tesla signale une évolution à la hausse (respectivement à la baisse) significativement plus forte que pour la moyenne des autres constructeurs. Plusieurs faits saillants peuvent ainsi être notés. Tout d'abord, le flux pour Tesla a dans un premier temps crû plus rapidement que pour la moyenne des autres constructeurs, puis à partir de 2012 a, au contraire, décrût plus rapidement. Ensuite, le point d'inflexion pour Tesla se situe en 2012, soit à la fois nettement plus tôt que pour la moyenne des autres constructeurs (pour lesquels il se situe en 2016 et est probablement purement «technique » du fait du délai entre dépôt et octroi des brevets) et nettement plus tôt que l'année 2014 marquée par l'annonce précitée de renoncement aux brevets par Tesla. L'antériorité de la baisse par rapport à l'annonce est encore plus marquée si on tient compte du fait que les brevets manquants, par rapport à une extrapolation à partir des années précédentes, auraient été déposés environ deux ans plus tôt compte tenu, une fois de plus, du délai moyen entre dépôt et octroi des brevets. Autrement dit, la baisse du flux d'inventions brevetées par Tesla daterait plutôt de 2010. Toutefois, la courbe de flux d'inventions nouvelles pour Tesla ne sort que rarement de la zone grisée, donc ne se démarque pas significativement de la dispersion des autres constructeurs autour du flux moyen. De fait, on observe bien une nette stabilisation du flux moyen pour les autres constructeurs à partir de 2013. Tesla serait donc plus proche du groupe de constructeurs dont le flux a assez fortement ralenti que de la moyenne, sans pour autant départir significativement de la moyenne des autres constructeurs. Sachant que peu d'autres constructeurs ont fait le même type d'annonce, ou alors en les restreignant à des domaines technologiques très spécifiques (tel Toyota sur l'hydrogène), on ne peut pas valider l'idée que l'annonce de 2014 marque un changement stratégique significatif de Tesla par rapport aux autres constructeurs. Cette conclusion est robuste à deux corrections.

La première correction apportée, tant à la Figure 3.a qu'à la Figure 3.b, est celle d'une consolidation des familles de Tesla Motors et SolarCity. Ce n'est certes qu'en 2016 que Tesla a racheté SolarCity mais les brevets déposés par SolarCity quelques années plus tôt, sous réserve qu'ils ont bien été renouvelés jusqu'en 2016, sont en conséquence tombés sous le contrôle de Tesla Motors. En outre, la coopération entre les deux firmes était déjà forte avant ce rachat, SolarCity annonçant dès 2009 mettre à disposition gratuitement des bornes de recharge pour les clients de Tesla. Les liens de parenté entre les dirigeants des deux entreprises, et le fait qu'Elon Musk siégeait au conseil d'administration de SolarCIty, n'y sont sans doute pas étrangers. Cette première correction ne change pas fondamentalement la donne; le point d'inflexion dans la dynamique restant en 2012. La seule différence est un pic ponctuel mais marqué de familles apportées par SolarCity en 2015, soit juste un an avant le rachat par Tesla et un an après l'annonce de renoncement aux brevets de Tesla. La seconde correction apportée concerne les classes technologiques considérées. Tesla ayant la particularité de concevoir et vendre exclusivement des véhicules électriques, son portefeuille est susceptible d'être centré sur les classes technologiques en lien avec les innovations sur ce type de véhicules alors que la plupart des autres constructeurs couvrent un spectre plus large de champs technologiques, incluant notamment ceux qui tiennent au moteur thermique. Or, il n'est pas impossible que la dynamique des brevets dépende en partie des champs technologiques. Afin de corriger ce potentiel biais, la Figure 3.b restreint l'analyse pour les

\footnotetext{
${ }^{11}$ La hauteur de la zone grisée correspond à l'écart type du flux de nouvelles familles pour les autres constructeurs que Tesla, centré sur le flux moyen.
} 
autres constructeurs que Tesla, ainsi que pour SolarCity, aux seules familles visant au moins un des champs technologiques ciblés par Tesla sur la période étudiée. A nouveau, hormis le pic ponctuel de nouvelles familles de SolarCity en 2015, l'analyse n'est pas fondamentalement affectée par cette correction.

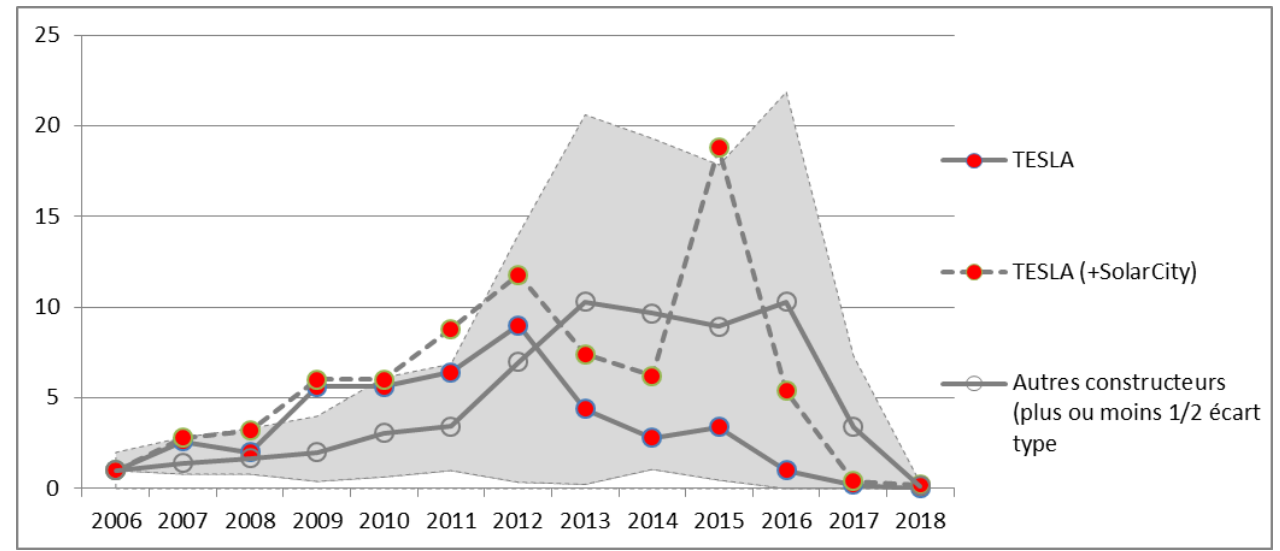

Figure 3.a. Flux de nouvelles familles de brevets de Tesla, pris isolément puis consolidé avec SolarCity, comparé à celui des autres producteurs (base 1 en 2006).

Source : auteurs à partir d'extractions de Patstat

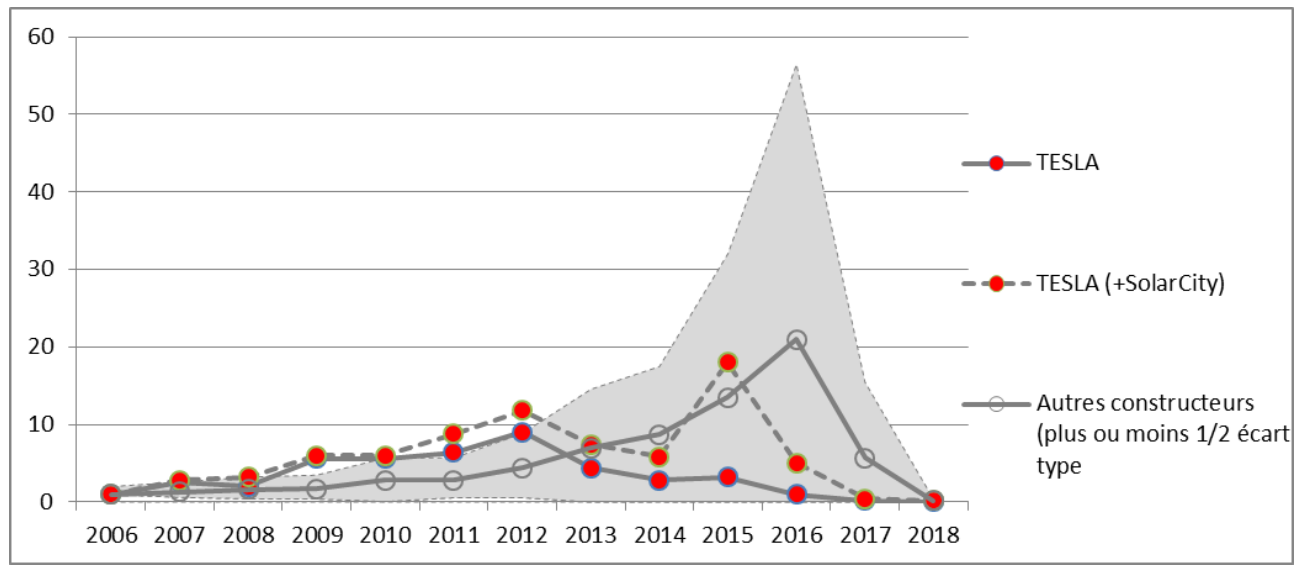

Figure 3.b. Flux de nouvelles familles de brevets de Tesla, pris isolément puis consolidé avec SolarCity, comparé à celui des autres producteurs (base 1 en 2006) en restreignant l'analyse aux seules familles ciblant au moins un des champs technologiques dans lesquels

Tesla a breveté sur la période.

Source : auteurs à partir d'extractions de Patstat

Le seul nombre de familles dans le portefeuille de brevets d'une firme ne renseigne qu'imparfaitement sur ce portefeuille, notamment sur la valeur économique des inventions brevetées. Une manière relativement simple d'avoir des éléments relatifs à cette valeur économique consiste à examiner en parallèle la taille des familles. En effet, plus une invention paraît prometteuse, plus la firme l'ayant obtenue sera encline à la protéger sur de nombreux marchés différents et, pour ce faire, à demander une extension du brevet initial, dit «priorité » auprès de nombreux autres offices de brevets. Typiquement, pour un constructeur visant à la fois les marchés nord-américain, européen et asiatiques (tout au moins le Japon, la Chine et la Corée), on s'attend donc à ce que la famille des brevets protégeant une même invention contienne un brevet USPTO (Etats Unis), un brevet EPO (Europe), un brevet JPO (Japon), un brevet CNIPA (Chine) et un brevet KIPO (Corée). Pour l'Europe, à défaut d'un brevet EPO il peut s'agir de brevets nationaux, 
notamment un brevet allemand auprès du DMPA et un français auprès de l'INPI. Suivant cette logique, la Figure 4 présente le taux de familles mono-brevet tandis que la Figure 5 présente la taille moyenne des familles, chaque fois en contrôlant du nombre de familles. Chacune de ces deux Figures présente les valeurs pour l'ensemble de la période couverte, soit de 2006 à 2018. Dans les deux cas, Tesla apparaît proche de la moyenne sur l'ensemble des constructeurs. Le nombre de familles ne semble être un déterminant majeur, ni de la proportion des familles mono-brevets, ni de la taille des familles. Sans grande surprise, la Figure 4 fait apparaître que les constructeurs pour lesquels la proportion de familles mono-brevets est la plus forte sont tous des constructeurs chinois n'ayant pas encore procédé à une internationalisation de leur marché, et donc absent notamment des marchés américains et européens. Geely fait exception, et de manière marquée, puisqu'il ressort au contraire comme le constructeur avec la plus faible proportion de familles mono-brevets. Il est à noter que Geely a la particularité d'être un constructeur privé, alors que ses compatriotes sont au moins en partie publics. De même, dès 2009 Geely a racheté Volvo et fait ainsi montre d'une volonté d'internationalisation. Ce fait saillant se retrouve à la Figure 5 où les constructeurs chinois se caractérisent par une taille moyenne des familles de brevets très proche de un, à l'exception de Geely qui caracole en tête de tous les constructeurs avec presque six brevets en moyenne par famille. Avec légèrement moins de deux brevets en moyenne par famille et un taux de familles mono-brevets de 0.6, Tesla Motors fait montre d'une internationalisation dans la protection de ses inventions proche de grands constructeurs japonais (Toyota, Honda), coréen (Hyundai), Allemands (Volkswagen, Audi, Daimler) ou Américains (GM, Chrysler) présents sur la plupart des marchés à travers le monde. Cette stratégie révèle les ambitions internationales de Tesla sans nécessairement le démarquer de la masse des autres constructeurs.

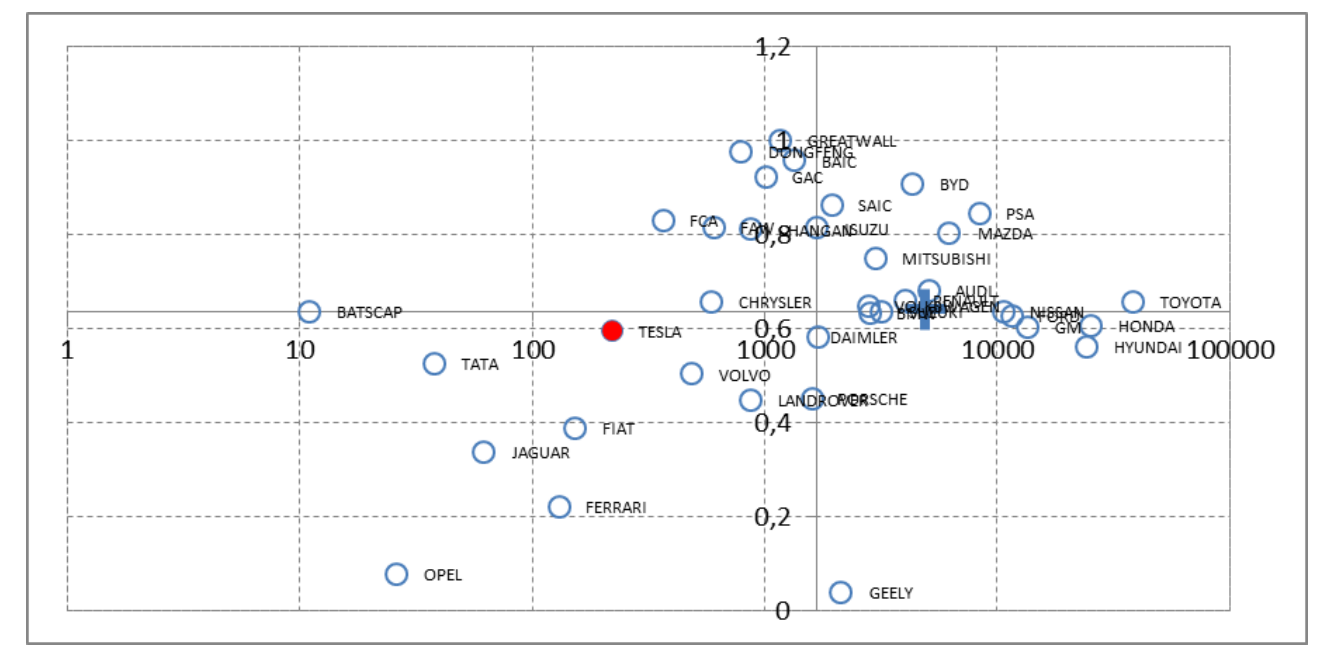

Figure 4. Taux de familles multi brevets en fonction de la taille du portefeuille de familles selon le constructeur (période 2006-2018, Tesla en rouge, graphe centré sur le point médian, le point moyen apparaissant en forme de croix, échelle horizontale logarithmique).

Source : auteurs à partir d'extractions de Patstat 


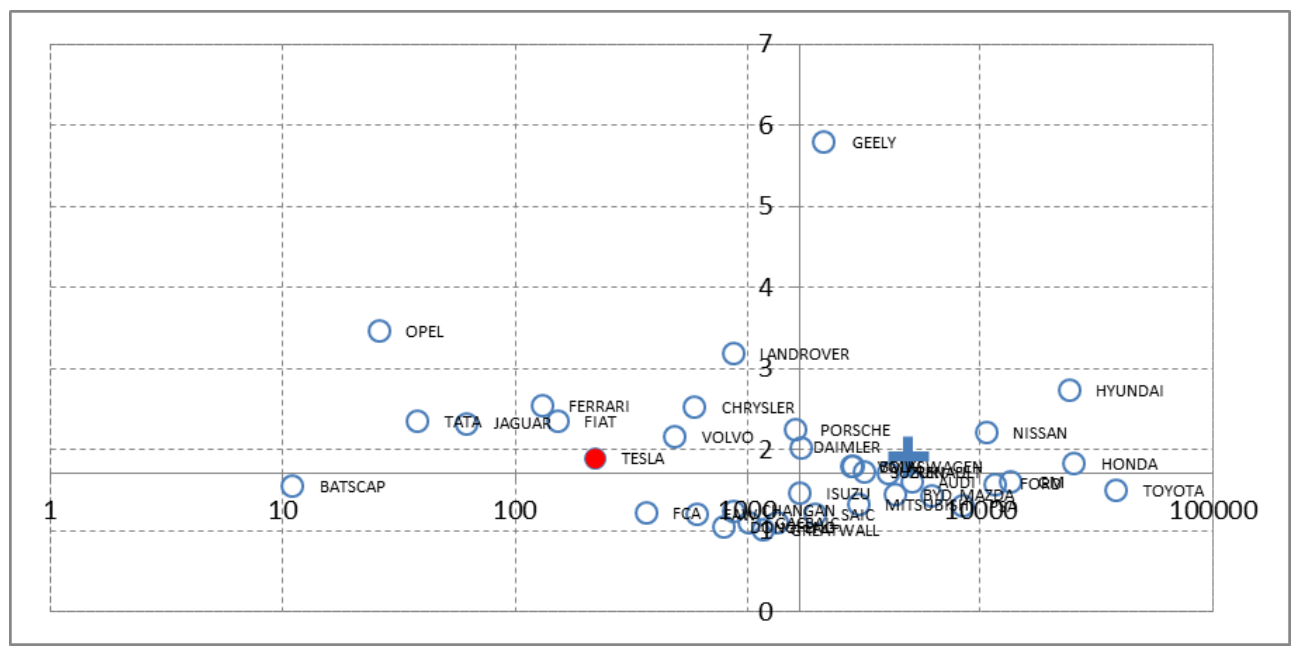

Figure 5. Taille moyenne des familles en fonction de la taille du portefeuille de familles selon le constructeur (période 2006-2018, Tesla en rouge, graphe centré sur le point médian, le point moyen apparaissant en forme de croix, échelle horizontale logarithmique).

Source : auteurs à partir d'extractions de Patstat

\section{A positionnement atypique, stratégie de coopération en R\&D atypique ?}

Les défis du développement de l'automobile à faibles émissions de carbone d'une part, du développement de l'automobile à conduite autonome d'autre part, nécessitent de la part des constructeurs des efforts conséquents de R\&D. Ils y répondent par des stratégies de mutualisation des moyens de R\&D. La première d'entre elles, qui n'est pas traitée ici, consiste en des opérations de fusions ou acquisitions. A titre d'exemple, le rachat d'Opel par PSA s'explique en partie par la faiblesse des investissements faits en R\&D par GM pour permettre à sa filiale européenne Opel de respecter les normes d'émissions de plus en plus restrictives fixées par l'Union Européenne, et par la nécessité pour PSA de rentabiliser ses propres investissements dans ce domaine par de plus grands volumes. Le rapprochement plus récent entre PSA et FCA obéit en partie à la même logique, couplée cette fois ci, avec une logique de diversification géographique. Une stratégie moins radicale de mutualisation des efforts de R\&D est la constitution d'une co-entreprise, ou joint-venture dans la terminologie anglaise, formalisant la coopération entre deux partenaires sur un sujet précis. C'est ainsi que le groupe Renault-Nissan a signé le 20 décembre 2018 un accord de joint-venture avec le chinois Jiangling Motors Corporation Group (JMCG) pour le développement de véhicules électriques en Chine ${ }^{12}$. De même Ford et Volkswagen ont annoncé en 2019 un projet de jointventure autour du véhicule électrique et du véhicule autonome. Une coopération de ce type sur un sujet de $R \& D$, tout particulièrement si le contrat de co-entreprise ne prévoit pas la constitution d'une entité disposant de la personnalité juridique, implique souvent le dépôt conjoint de brevets appelés dès lors co-brevets. De ce point de vue, analyser les co-brevets d'une entreprise peut être un moyen d'approcher ces coopérations de $R \& D$ en amont, c'est-à-dire sur des projets en phase de démarrage et où les partenaires et leurs compétences sont clairement identifiés par chacune des parties prenantes. Comme pour tout mécanisme de copropriété, le co-brevet nécessite un degré de confiance important entre partenaires. Même si les règles d'exercice de la copropriété de brevets peuvent fortement différer selon les offices, il en ressort toujours que les co-brevets présentent des risques importants en cas de désaccord des co-déposants ${ }^{13}$. Belderbos et alii [BEL 14], ou plus récemment

\footnotetext{
12 JMCG n'est pas répertorié dans les constructeurs traités par cette analyse car il s'agit d'un spécialiste des véhicules utilitaires et camions légers, mais pas des véhicules pour particuliers.

${ }^{13}$ Voir à ce sujet le rapport de 2017 de I'AIPPI "The Impact of Co-Ownership of Intellectual Property Rights on their Exploitation ", téléchargeable à l'adresse https://aippi.org/committee-publications/?committee-id=25536
} 
Lv Zeng et Lan [LV 18], ont examiné empiriquement les effets des co-brevets en termes de valeur crée et de valeur de l'entreprise. Par exemple, pour les co-brevets auprès de l'USPTO, chaque copropriétaire conserve sa totale liberté de cession de licence, voire de cession de sa part de brevet. Cela implique que ses partenaires peuvent se retrouver avec des bénéficiaires de licence ou de nouveaux copropriétaires qu'ils n'ont pas choisis et ceci indépendamment de leur volonté. A l'inverse, pour les co-brevets à l'Office Européen des Brevets (EBO) ou auprès de plusieurs offices nationaux européens, tout accord de licence ou de cession de parts de brevet requiert l'accord des autres copropriétaires. Chaque copropriétaire se trouve donc limité dans l'exercice de son droit. Le fait que, dans le cas d'une protection auprès de l'USPTO comme dans le cas d'une protection auprès de l'OEB, le co-brevet présente des risques non négligeables et a donc un «coût » par rapport à un brevet dont une firme serait unique propriétaire induit, du point de vue de l'analyse économique, que les co-brevets sont des signaux crédibles de la volonté de coopérer des co-déposants. En ce sens, comme discuté par Asay [ASA 16] dans un cadre plus général, les co-brevets véhiculent une information plus fiable, plus crédible et plus concrète qu'une simple annonce d'intention de coopérer pour appréhender la coopération en R\&D. C'est la raison pour laquelle ils sont utilisés par des auteurs tels que Goetze [GOE 10] pour étudier la coopération en R\&D.

La Figure 6 permet de se faire une idée de l'intensité de l'engagement des différents constructeurs dans des coopérations de $R \& D$ en amont. Elle croise deux informations complémentaires sur ce thème : la proportion de familles de brevets contenant au moins un co-brevet qui est portée sur l'axe horizontal et la proportion moyenne de co-déposants dans ces co-brevets qui est portée sur l'axe vertical. Il est à noter que les co-brevets traités ici ne se limitent pas à des co-brevets entre constructeurs. Ils intègrent tous les co-dépôts avec une autre entité que le constructeur en question, par exemple une structure de recherche académique ou un équipementier automobile. Positionné nettement en deçà de la médiane pour chacune de ces deux variables, Tesla Motors ressort à la Figure 6 comme un des constructeurs les moins engagés dans ce type de coopération de R\&D.

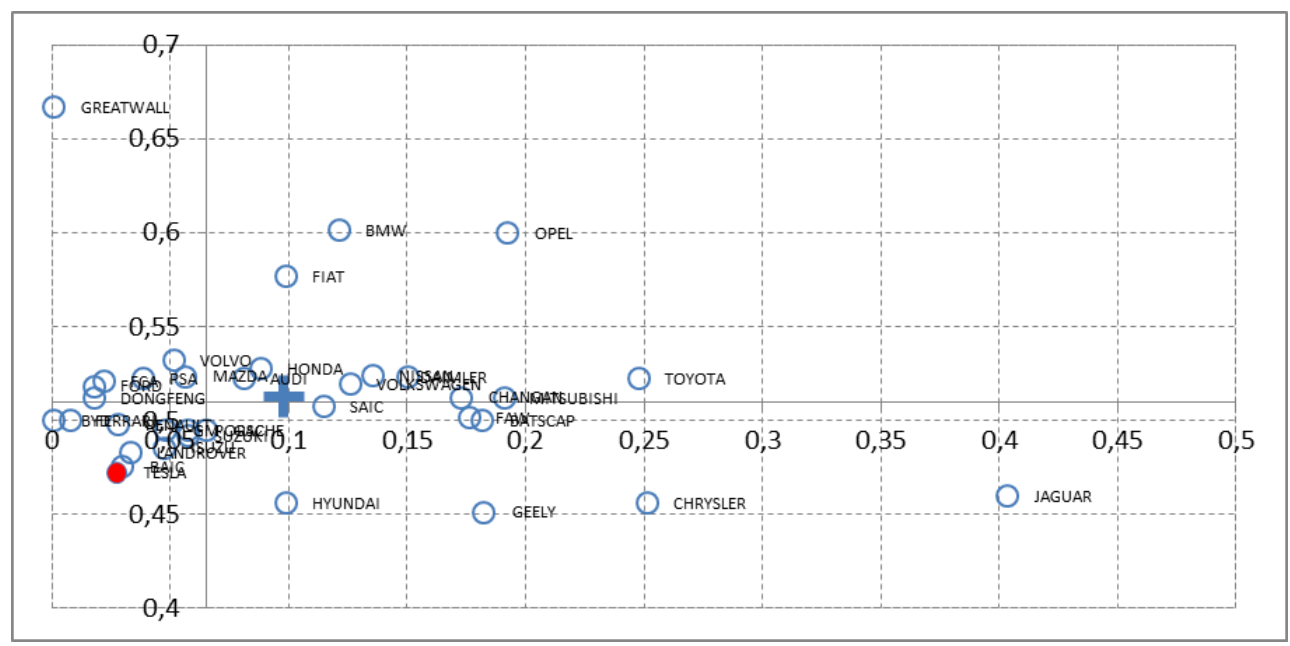

Figure 6. Positionnement des constructeurs en termes de taux de familles avec co-brevets -axe horizontalet de proportion moyenne de co-déposants dans les co-brevets -axe vertical-

(période 2006-2018, Tesla en rouge, graphe centré sur le point médian, le point moyen apparaissant en forme de croix). Source : auteurs à partir d'extractions de Patstat

La singularité de Tesla en matière de co-brevets peut faire écho à un positionnement atypique en matière d'innovation dans le domaine automobile. En effet, comme indiqué supra, les co-brevets sont surtout susceptibles d'apparaître dans le cas de coopération de R\&D où les partenaires et leurs compétences sont bien identifiés en amont. Cette identification en amont est plus aisée pour des projets qui s'inscrivent sur le sentier technologique dominant du secteur. A contrario, quand le 
projet sort des sentiers battus il peut beaucoup plus difficile d'apprécier l'importance des compétences et des connaissances apportées par les partenaires potentiels. Il n'est néanmoins pas simple de déterminer si une entreprise se positionne au cœur des développements technologiques novateurs de son secteur ou pas. Une possibilité consiste à s'appuyer sur l'analyse des réseaux de citations. Elle a été appliquée par exemple par Verspagen [VER 07] dans le cadre d'une étude de l'Histoire de la pile à combustible. Sa lourdeur de mise en œuvre tend toutefois à en limiter l'applicabilité à un secteur aussi large que le secteur automobile. La solution retenue dans ce qui suit s'appuie donc plutôt sur le concept de similitude technologique développé par Jaffe [JAF 86]. L'indice de similitude entre deux ensembles de brevets se calcule à partir des classes technologiques des brevets, plus exactement de la comparaison des fréquences de référence à ces classes entre les deux ensembles. Elle est indépendante du nombre de brevets contenus dans chaque ensemble. L'indice de similitude a typiquement été utilisé dans les travaux économétriques cherchant à estimer les effets de spillovers de R\&D entre entreprises; ces spillovers étant a priori d'autant plus importants entre deux entreprises que leurs portefeuilles de brevets présentent un indice de similitude élevé. L'indice de similitude est par construction compris entre zéro et un, et d'autant plus proche de un que la similitude entre les deux ensembles de brevets est élevée. Si l'indice de similitude est neutre à la taille du portefeuille, il n'est en revanche pas neutre de la calculer à partir des familles ou à partir des brevets. Dans le cas d'un calcul à partir des familles, chaque classe technologique ciblée par l'un ou l'autre des brevets de la famille ne se verra attribuer qu'une occurrence, indépendamment du nombre de brevets de la famille la ciblant. Dans le cas d'un calcul à partir des brevets, une classe technologique ciblée par plusieurs brevets d'une même famille se verra attribuer autant d'occurrences que de brevets de la famille la ciblant. Dans ce cas, l'indice de similitude aura donc tendance à surpondérer les classes technologiques des inventions protégées par une large famille de brevets. Sachant que la taille de la famille reflète en partie la valeur économique de l'invention, cela revient donc à corriger en partie la similitude de l'importance économique des inventions. C'est cette dernière approche qui a été retenue dans ce qui suit.

Deux mesures de similitude sont croisées à la Figure 7 afin de comprendre le positionnement de Tesla relativement aux autres constructeurs automobiles, toutes deux sont calculées à partir des classes technologiques CPC à quatre digits. La première mesure, portée sur l'axe horizontal, s'intéresse à la similitude entre le portefeuille de brevets de chaque constructeur et le portefeuille «moyen» regroupant tous les brevets de tous les constructeurs. Elle permet donc d'évaluer la singularité technologique d'un constructeur par rapporte à la moyenne des autres. Avec une valeur avoisinant les 0.68 pour cette première mesure, inférieure aussi bien à la médiane qu'à la moyenne, Tesla fait partie des constructeurs les plus éloignés du portefeuille «moyen » de brevets en termes de champs technologiques ciblés. Avec cette première mesure, le fait que deux constructeurs aient un indice de similitude prenant des valeurs proches n'implique toutefois pas que ces constructeurs sont par ailleurs similaires l'un à l'autre en terme technologique. Ils peuvent en effet avoir un éloignement d'ampleur comparable vis-à-vis du portefeuille «moyen », mais cet éloignement peut se faire dans des directions diamétralement opposées. C'est pourquoi une seconde mesure de similitude technologique est croisée à cette première mesure. Il s'agit de la similitude technologique de chaque constructeur par rapport au portefeuille de brevets de Tesla et en se limitant aux seuls brevets ciblant au moins une des classes ciblées par les brevets de Tesla. Par construction, l'indice de similitude de Tesla avec lui-même est de un, c'est-à-dire la valeur maximale possible. Les deux constructeurs qui, selon cette mesure, sont technologiquement les plus similaires à Tesla se révèlent être les deux constructeurs français Renault et PSA, suivis de constructeurs japonais (Honda, Mitsubishi), coréen (Huyndai) et chinois (Greatwall). Cela signifie que, si on ne regarde que les champs technologiques ciblés par Tesla, c'est pour ces six constructeurs que la répartition des brevets entre les différents champs technologiques ressemble le plus à la répartition caractérisant Tesla. Si certains de ces constructeurs (notamment Greatwall avec un des taux de familles avec cobrevets les plus faibles) apparaissaient aussi, comme Tesla, peu engagés dans la coopération de R\&D telle qu'appréhendée par les co-brevets, d'autres au contraire ont une intensité d'engagement (C) 2020 ISTE OpenScience - Published by ISTE Ltd. London, UK - openscience.fr

Page $\mid 12$ 
élevée (notamment Mitsubishi, Honda, Huyndai et, dans une moindre mesure, PSA). Ce n'est donc pas seulement le positionnement technologique de Tesla qui peut expliquer son faible engagement dans des coopérations menant à des co-brevets.

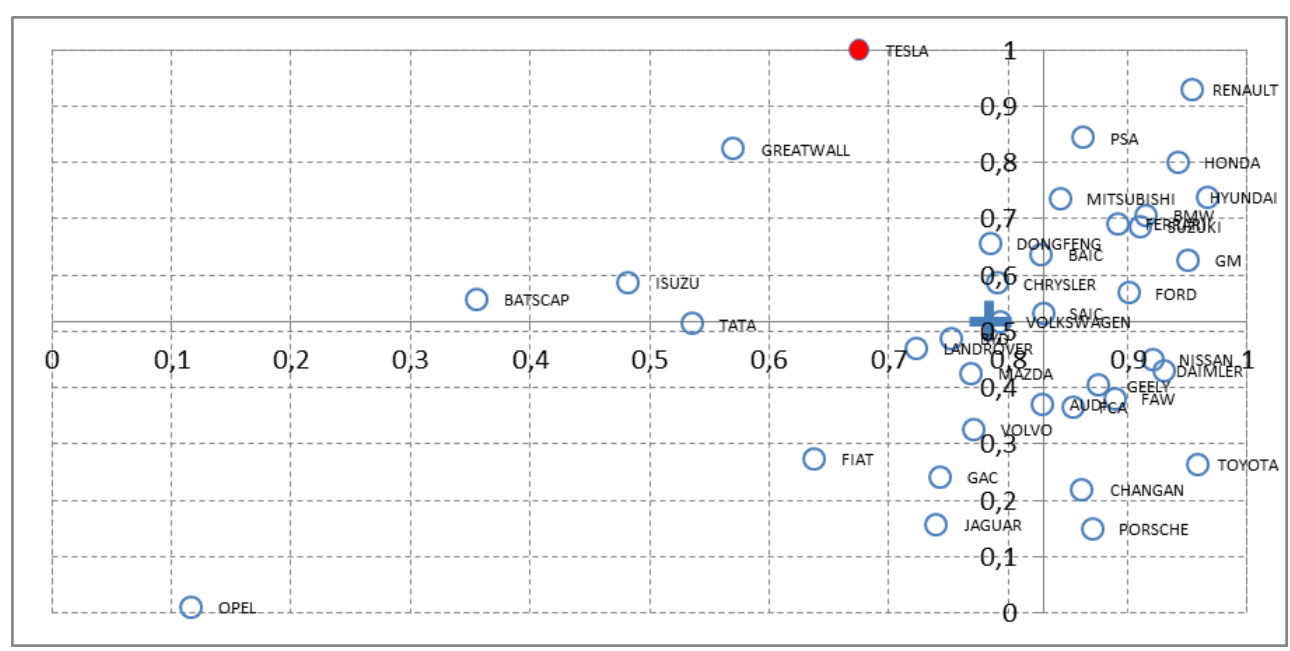

Figure 7. Similitude technologique des constructeurs au portefeuille moyen de brevets -axe horizontalet au portefeuille de Tesla pour les champs technologiques ciblés par Tesla -axe vertical-

(période 2006-2018, Tesla en rouge, graphe centré sur le point médian,

le point moyen apparaissant en forme de croix).

Source : auteurs à partir d'extractions de Patstat

\section{Patent pledge et leadership technologique}

Comme souligné supra dans la présentation des co-brevets, ces derniers révèlent des coopérations de R\&D pour lesquelles les partenaires se sont réciproquement identifiés et reconnus comme tels relativement en amont du processus d'innovation. La logique même de copropriété des brevets induit un traitement relativement symétrique des partenaires. Cette symétrie peut être corrigée contractuellement, par exemple au travers de clauses dans un accord de joint-venture définissant des clés de répartition du revenu des licences qui actent une contribution et donc une rétribution plus significative de l'un ou l'autre des partenaires. A contrario, le système des co-brevets, et plus généralement du joint-venture qui a pu y conduire, est peu adapté au cas d'une entreprise qui disposerait d'un leadership technologique et chercherait à entraîner d'autres acteurs derrière elle pour accroître les opportunités de développement de son invention. Dans ce cas, c'est plutôt une fois l'invention protégée par un ou plusieurs brevets que l'entreprise va chercher un effet de levier en adoptant une stratégie relevant des patent pledges.

La littérature, y compris académique, utilise le terme de patent pledge pour recouvrir des stratégies d'utilisation des brevets assez diverses. Néanmoins, toutes impliquent, à un certain degré, un affaiblissement dans l'exercice du droit d'exclusion par rapport à ce qui est légalement faisable. Ehrnsperger [EHR19] en propose une taxonomie à partir de la base de patent pledges constituée au College of Law de l'American University Washington ${ }^{14}$. Cela peut aller de licences FRAND, sur un ensemble de brevets assez circonscrit, au bénéfice d'autres détenteurs de brevets qui s'engagent dans un acte réciproque, à une liberté d'exploitation des brevets sans contrepartie financière ou non financière. Les patent pledges sont toutefois à distinguer du renoncement total au brevet, qui impliquerait que celui-ci tombe dans le domaine public. Le maintien du brevet permet en effet à son détenteur d'exercer une assertion défensive vis-à-vis de concurrents qui voudraient l'empêcher d'exploiter l'invention en arguant que cette exploitation serait en infraction avec leurs propres

\footnotetext{
${ }^{14}$ Accessible à l'adresse http://www.pijip.org/non-sdo-patent-commitments/
} 
brevets. Les motivations d'une firme pour se lancer dans un patent pledge peuvent être variées. Un élément ressort toutefois de manière assez récurrente [CON 15], [VER 17], [CON 19]: tirer parti d'externalités de réseau et d'effets d'échelle pour imposer une technologie ou un standard contrôlé par la firme. Vertinsky [VER 17] développe plus particulièrement cet argument dans le cas de Tesla.

Le développement du véhicule électrique repose tout d'abord sur des externalités de réseau importantes. En effet, l'achat d'un véhicule électrique est largement conditionné par le nombre et la répartition des bornes de recharge sur le territoire où le client prévoit de se déplacer. Parallèlement, la rentabilité de la constitution de ce réseau de bornes est d'autant plus forte qu'il y a un nombre important d'utilisateurs potentiels. Tout nouvel acquéreur d'un véhicule électrique sur un territoire donné renforce donc la rentabilité de mise en place du réseau et incite à son développement, au bénéfice des autres utilisateurs de véhicules électriques sur le même territoire. Il le fait sans en avoir conscience et sans en tenir compte dans sa décision. C'est cet effet qui est désigné sous le terme d'externalité de réseau [KAT 85]. L'intérêt de chaque constructeur de véhicules électriques est donc que ce type de véhicule se diffuse largement afin qu'un réseau de bornes conséquent soit mis en place. Il est intéressant de noter que cela n'a été le cas au tournant des XIX ${ }^{\text {ième }}$ et $X^{\text {ième }}$ siècles pour le développement des moteurs à essence. En effet, comme le détaille Melaina [MEL 07], l'essence était déjà vendue pour d'autres usages par divers magasins et c'est à partir de ces magasins que ce sont progressivement développées les stations modernes que nous connaissons. Autrement dit, le véhicule à essence n'a pas été confronté à l'absence initiale de système de distribution du carburant, lequel a néanmoins fortement évolué avec le développement de ce type de véhicule. Concernant Tesla, son intérêt pour le réseau de bornes de recharges électriques est accru par rapport aux autres firmes automobiles. En effet, comme pour celles-ci le véhicule tout électrique n'a qu'une importance marginale dans leur modèle d'affaire, elles ressentent moins urgemment le besoin du développement du réseau de bornes de recharge. Thomas et Maine [THO 19] considèrent même que ce développement peut aller contre leur intérêt au sens où il permettrait au véhicule électrique de s'imposer et rendrait trop rapidement obsolète l'ensemble des actifs matériels et immatériels liés au véhicule à moteur thermique dans lesquels ils ont lourdement investi. Ces arguments sont confortés par l'observation que Tesla a, quant à lui, investi dans l'ensemble des maillons clés du véhicule électrique et notamment dans les bornes de recharge et les mécanismes de production d'électricité à partir d'énergie renouvelable avec le powerwall. Tesla dispose donc d'un système "prêt à l'emploi » standardisé par rapport à ses produits. Son intérêt est en outre que les concurrents s'appuient sur son standard plutôt que de les voir mettre en place les leurs. A ces effets de réseaux s'ajoutent des effets d'échelle. Ils concernent surtout la production des batteries pour laquelle on tend à considérer que des baisses de coûts importants peuvent être escomptées de l'augmentation marquée des volumes de production. C'est dans cet esprit que Tesla a investi dans sa première Gigafactory, de même qu'un certain nombre de fabricants asiatiques de batteries, et que de son côté l'Union Européenne tente de mettre en place un "Airbus des batteries ». Là encore, l'intérêt de Tesla est d'amener le plus grand nombre de constructeurs possible à préférer lui acheter ses batteries plutôt que de fabriquer les leurs, ou les acheter à d'autres fabricants. On notera enfin qu'un problème similaire se pose pour le développement du véhicule à hydrogène. Or, c'est précisément sur cette technologie que l'autre patent pledge bien connu du secteur automobile porte, celui de Toyota. Pour que les patent pledges fondés sur les économies de réseau et les économies d'échelle fonctionnent, encore faut-il que la technologie ainsi ouverte à la concurrence soit convaincante, autrement dit que la firme pratiquant le patent pledge dispose d'un réel leadership technologique, à défaut de disposer au moment du patent pledge d'un leadership sur le marché.

Afin de tester le leadership de Tesla sur les solutions technologiques qu'il ouvre à ses concurrents, il est possible de s'appuyer sur l'analyse des citations reçues par les brevets ainsi mis à disposition des concurrents par le patent pledge. Plus exactement, il s'agit d'évaluer si les brevets de Tesla sont significativement plus cités par ses concurrents, que les brevets détenus par ces concurrents et ciblant les mêmes domaines technologiques. Il convient donc de ne regarder que les 
«non self citations », c'est à dire les citations faites par d'autres que le constructeur détenant les brevets considérés. De même, il convient pour les concurrents de Tesla de restreindre l'analyse aux citations reçues par les seuls brevets ciblant les mêmes classes technologiques que celles ciblées par Tesla. C'est ce que fait la Figure 8, en complétant par la construction d'un couloir grisé correspondant pour chaque âge des brevets à la moyenne augmentée ou diminuée d'un demi écart type du décompte de citations reçues par les brevets de cet âge détenus par les concurrents de Tesla. Ce couloir grisé indique donc la variabilité standard du décompte de citations reçues par les brevets des concurrents. Or, le décompte moyen des citations reçues par les brevets de Tesla est nettement en dehors, et au-dessus, de ce couloir. Afin de confirmer ce résultat, la Figure 8 fait également apparaître le maximum sur les autres constructeurs du nombre moyen de citations reçues par brevet de leur portefeuille. Hormis ponctuellement pour quelques âges, le nombre moyen de citations pour les brevets de Tesla excède ce maximum. La Figure 8 met donc clairement en exergue que les brevets de Tesla sont nettement plus cités que ceux de ses concurrents. En ce sens, la Figure 8 confirme le leadership technologique de Tesla. Si Tesla n'apparaît pas comme un gros détenteur de brevets selon les éléments qui précèdent, en revanche les brevets qu'il détient sont bien considérés comme des brevets importants par ses concurrents au vu des citations reçues. Tesla surpasse même nettement ses concurrents du secteur des constructeurs d'automobiles en la matière. Les résultats de cette analyse confortent donc l'idée que le patent pledge de Tesla vise à exploiter son leadership technologique pour le transformer en leadership sur le marché.

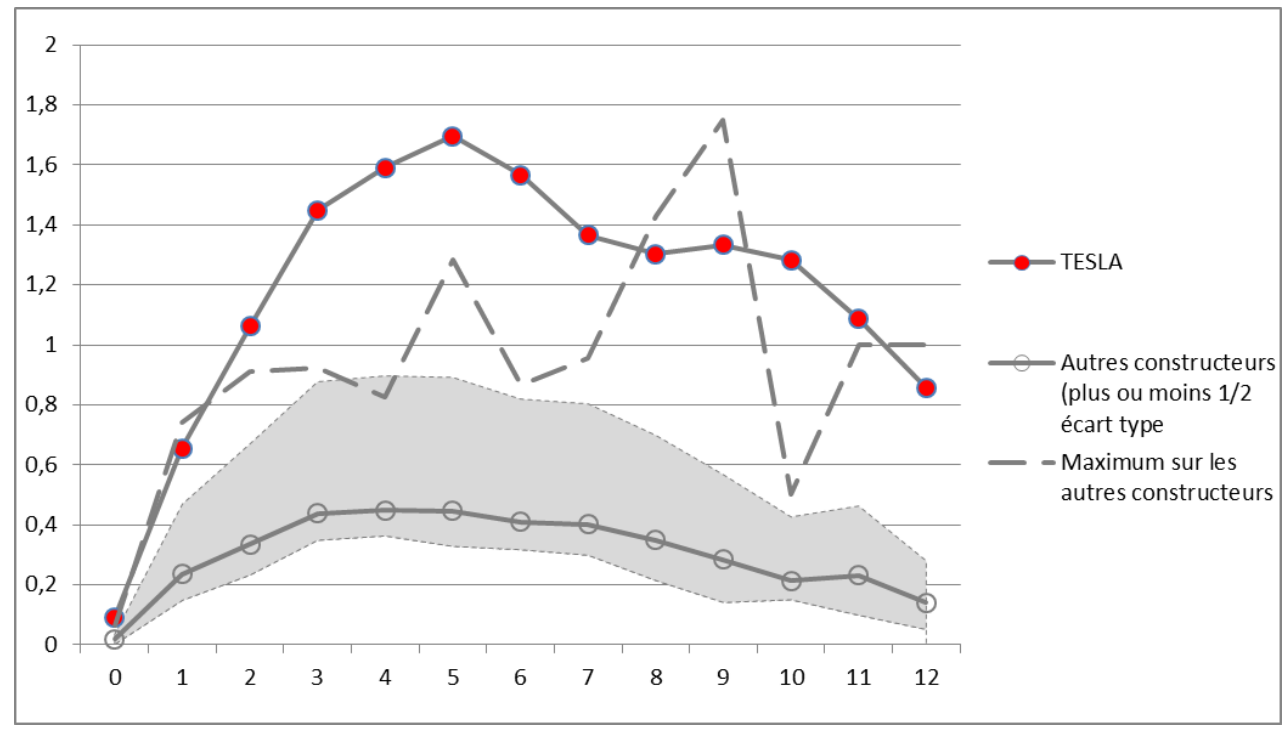

Figure 8. Profil des citations " non self » reçues par famille en fonction de l'âge, pour Tesla et en moyenne sur les autres constructeurs, pour les seules classes technologiques ciblées par Tesla (période 2006-2018).

Source : auteurs à partir d'extractions de Patstat

\section{Conclusion}

Le patent pledge de l'entreprise Tesla est un cas d'étude particulièrement intéressant. Il correspond en effet à une stratégie d'ouverture de son portefeuille de brevets par une entreprise nouvelle, qui a investi dans un secteur déjà fortement concentré en ciblant un marché spécifique, celui du véhicule tout électrique, par le haut de gamme. Si Tesla est en bonne position en termes de ventes sur ce marché spécifique, les constructeurs automobiles traditionnels ont également développé leur offre et déposent aujourd'hui nettement plus de brevets que Tesla lui-même sur les classes technologiques ciblées par ce dernier. Il ressort toutefois de l'analyse des brevets que Tesla se singularise. Tesla est tout à la fois très peu engagé dans les coopérations de R\&D en amont, au moins tel qu'il est possible d'en juger par les co-brevets de la firme en comparaison avec ceux de ses concurrents, et au cœur des classes technologiques où il brevète à en juger par le nombre 
significativement plus élevé de citations reçues par ses brevets. Ces éléments arguent en faveur d'une interprétation en termes de recherche de leadership technologique par Tesla. Cette interprétation est confortée par l'intégration verticale de Tesla qui propose aussi des systèmes de recharge et des systèmes de stockage individuel de l'électricité. Selon cette interprétation, le patent pledge est un moyen d'amener les concurrents à s'aligner sur les solutions techniques proposées par Tesla afin pour ce dernier de tirer parti d'externalité de réseau et d'économies d'échelle et de baisser ainsi ses coûts pour imposer sa technologie sur le marché des véhicules tout électriques.

\section{Références}

[ASA 6] ASAY, C.D., " The informational value of patents », vol. 31, Berkeley Technology Law Journal, p. 260-324, 2016.

[BAR 14] BARMORE, C., 2014, "Tesla unplugged: automobile franchise laws and the threat to the electric vehicule market », vol. 18, n², Virginia Journal of Law \& Technology, p. 185-228, 2014.

[Bel 14] Belderbos, R., Cassiman, B., Faems, D., Leten, B., VAN Looy, B., " Co-ownership of intellectual property: Exploring the value-appropriation and value-creation implications of co-patenting with different partners », vol. 43, Research Policy, p. 841-852, 2014.

[CON 15] CONTRERAS, J. L., " Patent pledges », vol. 47, n³, Arizona State Law Journal, p. 543-608, 2015.

[CON 19] Contreras, J. L., Hall, B. H. \& C. Helmers, « Pledging patents for the public good: rise and fall of the eco-patent commons », vol. 57, n¹, Houston Law Review, p. 61-109, 2019.

[CRA 16] CRANE, D. A., "Tesla, dealer franchise law, and the politics of crony capitalism », vol. 101, Iowa Law Review, p. 572-607, 2016.

[HER 19] EHRnsPerger, J. F., TIETZE, F., « Patent pledges, open IP, or patent pools? Developping taxonomies in the thicket of terminologies », vol. 14, n 8, PLoS ONE, p. 1-18, 2019.

[GOE 10] GoETZE, C., " An empirical enquiry into co-patent networks and their stars: the case of cardiac pacemaker technology », vol. 30, Technovation, p. 436-446, 2010.

[JAF 86] JAFFE, A. B., " Technological Opportunity and Spillovers of R\&D: Evidence from Firms' Patents, Profits, and Market Value », vol. 76, n5, American Economic Review, p. 984-1001, 1986.

[KAT 85] KATZ, M. L., ShaPIRo, C., « Network Externalities, Competition, and Compatibility, vol. 75, n³, American Economic Review, p. 424-440, 1985.

[LV 18] LV, D. D., ZENG, P., LAN, H., 2018, « Co-patent, financing constraints, and innovation in SMEs: an empirical analysis using market value panel data of listed firms", vol. 48, Journal of Engineering and Technology Management, p. 15-27, 2018.

[MEL 07] MeLAINA, M. W., 2007, « Turn of the century refueling: A review of innovations in early gasoline refueling methods and analogies for hydrogen », vol. 35, Energy Policy, p. 4919-4934, 2007.

[STO 15] STOLZE, E., " A billion dollar franchise fee? Tesla motors' battle for direct sales: state dealer franchise law and politics ", vol. 34, n³, Franchise Law Journal, p. 293-309, 2015.

[THO 19] Thomas, V. J., MAINE, E., " Market entry strategies for electric vehicule start-ups in the automotive industry - lessons from Tesla Motors », vol. 235, Journal of Cleaner Production, p. 653-663, 2019.

[VER 07] Verspagen, B., " Mapping Technological Trajectories as Patent Citation Networks: a Study on the History of Fuel Cell Research ", Advances in Complex Systems, vol. 10, p. 93-115, 2007.

[VER 17] VERTNISKY, L., « Hidden costs of free patents », vol. 78, n6, Ohio State Law Journal, p. 1379-1448, 2017. 